\title{
DZWIR 2012/8
}

\section{AUFSÄTZE}

Markus Geißler

Zur Gültigkeit gläubigerbeschwerender Abfindungsklauseln bei der Pfändung in GmbH-Anteile und der Gesellschafterinsolvenz 311 Rainer Smola

Einfluss des Wahlrechts des Insolvenzverwalters nach $\S 103$ InsO auf die Aussonderungsfähigkeit nach $§ 22 \mathrm{j}$ KWG (Refinanzierungsregister) 318

Nils Dietrich/Stephan Szalai

Die künftige Regelung des Umfangs der Nacherfüllung im Kaufrecht Folgen des BGH-Urteils vom 21. 12. 2011 - VIII ZR 70/08 319

\section{RECHTSPRECHUNG}

FINANZGERICHTSBARKEIT

BFH, Urteil vom 30.11.2011 - I R 100/10,

Keine Passivierung bei sog. qualifiziertem Rangrücktritt 327

BFH, Urteil vom 14. 12. 2011 - I R 72/10,

Passivierung »angeschaffter« Rückstellungen bei steuerlichem

Ausweisverbot 329

ZIVILGERICHTSBARKEIT

BGH, Urteil vom 21. 7.2011 - IX ZR 120/10,

Hausgeldansprüche in der Insolvenz eines Wohnungseigentümers 331

BGH, Urteil vom 13. 10. 2011 - IX ZR 188/10,

Aufhebung der Zwangsverwaltung nach Antragsrücknahme 334

BGH, Beschluss vom 1.12.2011 - IX ZB 260/10

Versagung der Restschuldbefreiung bei unrichtigen oder unvollständigen Kreditangaben 337
BGH, Urteil vom 1.12. 2011 - IX ZR 58/11,

Lastschrifteinziehung eines wiederkehrenden Sozialversicherungsbeitrags 339

BGH, Urteil vom 8. 12. 2011 - IX ZR 33/11,

Anfechtung der Übereignung eines in Deutschland belegenen Grundstücks 341

BGH, Urteil vom 24.1.2012 - II ZR 109/11,

Wirksamwerden eines Einziehungsbeschlusses

(Anmerkung Tilo Keil) 346

BGH, Beschluss vom 9. 2. 2012 - IX ZB 86/10,

Bedingter Eigenantrag auf Eröffnung des Insolvenzverfahrens 350

BGH, Beschluss vom 9. 2.2012 - IX ZB 248/11,

Beschwerde des Schuldners gegen die Eröffnung des Insolvenz-

verfahrens 351

BGH, Beschluss vom 15.3.2012 - IX ZA 107/11,

Anfechtung einer Nichtauszahlung von Aufwendungshilfen zur Wohnbauförderung 352

BGH, Beschluss vom 26.4.2012 - IX ZB 273/11,

Herausgabevollstreckung aus einem Insolvenzeröffnungsbeschluss 353

OLG Nürnberg, Urteil vom 9. 1.2012 - 4 U 931/11,

Zurechnung von Kenntnissen über drohende Zahlungsunfähigkeit eines Schuldners innerhalb verschiedener Stellen eines Finanzamts 353

\section{VERANSTALTUNGEN}

\author{
Arbeitsgemeinschaft Handels- und Gesellschaftsrecht im DAV/ \\ DeutscheAnwaltakademie \\ »7. Deutscher Handels- und Gesellschaftsrechtstag « \\ - Aktuelles aus der Gesetzgebung (MinR Prof. Dr. Ulrich Seibert, BMJ, \\ Berlin); Aktuelles aus der Rechtsprechung des BGH (VorsRiBGH \\ Dr. Alfred Bergmann, Karlsruhe); Der Ausschluss aus der GmbH - \\ von der Beschlussfassung bis zur Abfindung, Satzungsklausel (RA \\ Dr. Thomas Trölitzsch, Stuttgart); Schenkung zwischen Gesellschaft \\ und Gesellschafter (RA Prof. Dr. Burkhard Binnewies, Köln); Um- \\ wandlungen nach dem Umwandlungssteuererlass (RA Dr. Rolf \\ Schwedhelm, Köln); Kapitalkonten in Personengesellschaftsverträ- \\ gen (Notar Dr. Eckhard Wälzholz, Füssen) \\ - 14. 9. 2012, 9:30 h bis 15. 9. 2012, 13:00 h; Pullman Berlin Schwei- \\ zerhof; Preis: $350,-€ / 400,-€ / 450,-€$ zzgl. USt. \\ - www.anwaltakademie.de
}

\section{Arbeitsgemeinschaft Verkehrsrecht im DAV/Deutsche Anwalt- akademie \\ »Grenzüberschreitende Schadenregulierung « \\ - Prof. Dr. Ansgar Staudinger, Univ. Bielefeld \\ - 22. 9. 2012, 9:30 h-17:00 h; Neuss; Preis: $160,-€ / 249,-€ / 274,-€$ zzgl. USt. \\ - www.anwaltakademie.de}

Berlin/Brandenburger Arbeitskreis für Insolvenzrecht e. V.

- Vortrag Prof. Dr. Stefan Smid, Univ. Kiel

- 6. 9. 2012, 18:00 h, IHK, Kleiner Vortragssaal UG, Fasanenstraße 85, 10623 Berlin

- www.insolvenzverein.de
Institut für Insolvenzrecht e. V.

»Best of BGH: Aktuelle Rechtsprechung des BGH «

- RiBGH Gerhard Vill, Karlsruhe

- 24. 9. 2012, 17:00 h, Dormero Hotel, Hannover

- www.institut-insolvenzrecht.de

\section{Münchener Fachkolleg für Insolvenzrecht}

"6 Monate ESUG - erste (positive?) Zwischenbilanz aus Sicht eines Insolvenzrichters, eines Verwalters und eines Bankenvertreters"

- RiAG Prof. Dr. Heinz Vallender, Köln; RA Dr. Jürgen Blersch, Wiesbaden; RA Ralf Zuleger, München

- 14. 9. 2012, 10:30 h-16:45 h, Hotel Best Western NH Delta Park, 68165 Mannheim

- Preis: 495,- € zzgl. USt.; www.mfinso.de

Wustrauer Arbeitskreis Insolvenzrecht e. V.

»Sanierungschancen und Haftungsrisiken beim Insolvenzplan in der Eigenverwaltung "

- RA Dr. Jürgen D. Spliedt, Berlin

- 21.9. 2012, 10:00 h, Maritim Hotel Berlin, Stauffenbergstraße 26, 10785 Berlin

- Tel. 0331-275900 


\section{NACHRICHTEN}

\section{BAG, Fehler bei der Erstattung einer Massenentlassungsanzeige}

Begeht der Arbeitgeber bei der Erstattung einer nach $\$ 17$ KSchG erforderlichen Massenentlassungsanzeige Fehler, werden diese durch einen bestandskräftigen Bescheid der Agentur für Arbeit nach $\S \S 18,20$ KSchG nicht geheilt. Die Arbeitsgerichte sind durch einen solchen Bescheid nicht gehindert, die Unwirksamkeit der Massenentlassungsanzeige festzustellen - so das Bundesarbeitsgericht mit Urteil vom 28. 6. 2012 - 6 AZR 780/10.

Der Kläger war seit 1990 bei der Schuldnerin beschäftigt. Am 1.3. 2009 wurde über das Vermögen der Schuldnerin das Insolvenzverfahren eröffnet und der Beklagte zum Insolvenzverwalter bestellt. Auf der Grundlage eines noch während des vorläufigen Insolvenzverfahrens mit seiner Zustimmung geschlossenen Interessenausgleichs mit Namensliste vom 24.2. 2009 kündigte der Beklagte am 11.3. 2009 das Arbeitsverhältnis zum 30.6.2009. Am 26.2. 2009 hatte die Schuldnerin Massenentlassungsanzeige bei der Agentur für Arbeit erstattet, ohne den Interessenausgleich beizufügen. Der Anzeige war entgegen der gesetzlichen Anordnung in $\S 17$ Abs. 3 Satz 2 KSchG auch keine Stellungnahme des Betriebsrats beigefügt. Der Betriebsrat der Schuldnerin erklärte am 26. 2. 2009 allerdings schriftlich gegenüber der Agentur für Arbeit, er sei darüber informiert, dass eine Massenentlassungsanzeige abgesandt worden sei. Noch am 26. 2. 2006 bestätigte die Agentur für Arbeit den Eingang der Massenentlassungsanzeige. Später verkürzte sie die Sperrfrist. Der Kläger hat die Kündigung - in allen Instanzen erfolgreich - angegriffen.

Die Beifügung der Stellungnahme des Betriebsrats, ersatzweise des Interessenausgleichs mit Namensliste, ist Voraussetzung für eine wirksame Massenentlassungsanzeige. Das Schreiben des Betriebsrats vom 26. 2. 2009 an die Agentur für Arbeit enthielt keine eindeutige, abschließende Meinungsäußerung zu den angezeigten Kündigungen und war deshalb keine ordnungsgemäße Stellungnahme im Sinne von § 17 Abs. 3 Satz 2 KSchG. Der Bescheid der Agentur für Arbeit über die Verkürzung der Sperrfrist hat den Formfehlernichtgeheilt. Die Wirksamkeit der Massenentlassungsanzeige ist von der Bindungswirkung eines solchen Bescheids nicht umfasst.

\section{BGH, Haftung von Gründungsgesellschaftern}

Gründungsgesellschafter haften gemäß Urteil des BGH vom 23. 4. 2012 - II ZR 211/09 dem über einen Treuhänder beitretenden Anleger auf Schadensersatz aus Prospekthaftung im weiteren Sinne, wenn der Treugeber nach dem Gesellschaftsvertrag wie ein unmittelbar beitretender Gesellschafter behandelt werden soll. Nach weiterem Urteil vom 14. 5.2012 - II ZR 69/12 haftet ein Gründungsgesellschafter, der sich zu den vertraglichen Verhandlungen über den Beitritt eines Anlegers zu einer Fondsgesellschaft eines Vertriebs bedient und diesem oder von diesem eingeschalteten Untervermittlern die geschuldete Aufklärung der Beitrittsinteressenten überlässt, für deren unrichtige oder unzureichende Angaben.
BGH, Veräußerung eines Warenlagers mit Zustimmung der Bank und Treuhandvereinbarung

Veräußert ein Schuldner mit Zustimmung seiner Bank ein in deren Sicherungseigentum stehendes Warenlager mit der treuhänderischen Vereinbarung, dass der Kaufpreis auf das bei dieser Bank im Soll geführte Kontokorrentkonto des Schuldners zu zahlen ist, so benachteiligt die Verrechnung der Gutschriften aus den Kaufpreisen mit Gegenforderungen der Bank die Gläubiger in Höhe des Wertes des aufgegebenen Sicherungseigentums nicht (BGH, Urteil vom 26. 4. 2012 - IX ZR 67/09); der Wert des Sicherungsguts ist mit dem für den Warenbestand erzielten Kaufpreis zu bemessen, wenn dieser hinter dem Einkaufswert zurückbleibt.

\section{BGH, Mittelbare objektive Gläubigerbenachteiligung}

Eine mittelbare objektive Gläubigerbenachteiligung ist nach dem Urteil des Bundesgerichtshofs vom 26.4.2012 - IX ZR 146/11 gegeben, wenn durch die angefochtene Rechtshandlung eine Forderung des Anfechtungsgegners, die im Fall der Eröffnung des Insolvenzverfahrens eine bloße Insolvenzforderung gewesen wäre, zur Masseverbindlichkeit aufgewertet wird. Eine Vertragsübernahme kann als unentgeltliche Leistung anfechtbar sein. Ob eine Vertragsübernahme unentgeltlich ist, ist grundsätzlich nach dem Verhältnis von Leistung und Gegenleistung in dem übernommenen Vertrag zu beurteilen.

\section{$\mathrm{BGH}$, Rechtsbeschwerde in Insolvenzsachen}

Nach der Aufhebung des $§ 7$ InsO durch das Gesetz zur Änderung des $\$ 522$ der Zivilprozessordnung findet die Rechtsbeschwerde in Insolvenzsachen nur gegen solche Beschwerdeentscheidungen zulassungsfrei statt, die vor dem Inkrafttreten des neuen Rechts am 27.10.2011 erlassen worden sind (BGH, Beschluss vom 10.5.2012 - IX ZB 295/11; Bestätigung des Senatsbeschlusses vom 20.12.2011 - IX ZB 294/11). Die Entscheidung über die Zulassung der Rechtsbeschwerde kann nicht vom Rechtsbeschwerdegericht nachgeholt werden, wenn das Beschwerdegericht verkannt hat, dass ihm diese Entscheidung oblegen hat (Bestätigung der Senatsentscheidung vom 24.7.2003 - IX ZB 539/02, DZWIR 2004, 72 mit Anm. C. W. Hergenröder).

$\mathrm{BGH}$, Herausgabe gezogener Nutzungen aus wirksam angefochtenen Steuerzahlungen

Nach dem Urteil des BGH vom 24. 5. 2012 - IX ZR 125/11 ist der Fiskus von der Rechtshandlung an dem Insolvenzverwalter zur Herausgabe gezogener Nutzungen aus wirksam angefochtenen Steuerzahlungen verpflichtet, wobei es auf die steuerliche Ertragshoheit nicht ankommt. Als gezogene Nutzungen herauszugeben sind Zinserträge von Einnahmeüberschüssen, die im Haushaltsvollzug ausnahmsweise zeitweilig nicht benötigt werden, und ersparte Zinsen für Kassenverstärkungskredite oder andere staatliche Refinanzierungsinstrumente, die infolge des Eingangs wirksam angefochtener Steuerzahlungen zurückgeführt oder vermieden worden sind.

\section{IMPRESSUM}

Der Verlag hat für die Wiedergabe aller in dieser Zeitschrift enthaltenen Informationen (Programme, Verfahren, Mengen, Dosierungen, Applikationen etc.) mit Autoren und Herausgebern große Mühe darauf verwandt, diese Angaben genau entsprechend dem Wissensstand bei Fertigstellung des Werkes abzudrucken. Trotz sorgfältiger Herstellung des Manuskripts und Korrektur des Satzes können Fehler nicht ganz ausgeschlossen werden. Autoren bzw. Herausgeber und Verlag übernehmen infolgedessen keine Verantwortung und keine daraus folgende oder sonstige Haftung, die auf irgendeine Art aus der Benutzung der in dem Werk enthaltenen Informationen oder Teilen davon entsteht. Die Wiedergabe von Gebrauchsnamen, Handelsnamen, Warenbezeichnungen und dergleichen in dieser Zeitschrift berechtigt nicht zu der Annahme, dass solche Namen ohne weiteres von jedermann benutzt werden dürfen. Vielmehr handelt es sich häufig um gesetzlich geschützte, eingetragene Warenzeichen, auch wenn sie nicht eigens als solche gekennzeichnet sind.

ISSN 1439-1589 e-ISSN 1612-7056

SCHRIFTLEITER Rechtsanwalt Michael Schmidt, Kurfürstendamm 171, D-10707 Berlin, Tel.: +49 (0)308857 90-0, Fax: +49 (0)30885790-10, Email: m.schmidt@mac.com

JOURNAL MANAGER Christiane Geißler, De Gruyter, Genthiner Straße 13, 10785 Berlin, Germany, Tel.: +49 (0)30 $26005-260$, Fax: +49(0)30260 05-250,

Email: christiane.geissler@degruyter.com

ANZEIGENVERANTWORTLICHE Panagiota Herbrand, De Gruyter, Mies-van-derRohe-Straße 1, 80807 München, Germany, Tel.: +49 (0)89769 02-394 Fax:+49(0)89769 02-350, Email: panagiota.herbrand@degruyter.com (C) 2012 Walter de Gruyter GmbH \& Co. KG, Berlin/Boston A 20288

SATZ jürgen ullrich typosatz, Nördlingen DRUCK Franz X. Stückle Druck und Verlag e. K., Ettenheim Printed in Germany

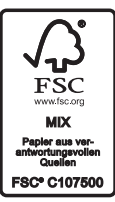

